\title{
ESTABLECIMIENTO AGROPRODUCTIVO ECOLOGICO VS. AGRICULTURA CONVENCIONAL: PARTIDO DE TANDIL, PROVINCIA DE BUENOS AIRES
}

\section{Organic agroproductive facility vs. Conventional agriculture: Tandil county, Buenos Aires province}

Patricia Vasquez Universidad Nacional del Centro de la Provincia de Buenos Aires, Buenos Aires, Argentina patriciavazquez11@gmail.com

Milagros Vignolles

Universidad Nacional del Centro de la Provincia de Buenos Aires, Buenos Aires, Argentina milagrosvignolles@hotmail.com

Artigo recebido em 07/08/2014 e aceito para publicação em 06/07/2015

RESUMEN: En Argentina, en las últimas décadas, se ha generado un proceso denominado como "agriculturización", con diversos impactos ambientales. Lo anterior ha llevado a plantear modelos agrícolas más sustentables. El presente trabajo propone analizar la sustentabilidad de un establecimiento rural dedicado a la agricultura ecológica con respeto a los sistemas agrícolas convencionales, ubicados en el partido de Tandil, provincia de Buenos Aires.

La metodología aplicada se basa en el uso de indicadores de sustentabilidad, trabajos antecedentes, entrevistas, matriz de debilidades-amenazas-fortalezas-oportunidades, y propuestas de corrección de puntos críticos. Se observa que el establecimiento con prácticas ecológicas, a diferencia de aquellos que utilizan prácticas convencionales, presenta múltiples beneficios tales como alta biodiversidad, diversificación de actividades, cultivos orgánicos, autoabastecimiento. Los puntos críticos se ven reflejados en las amenazas y debilidades del sistema, tales como el manejo del suelo para la siembra y la productividad media en cultivos, siendo menor a los sistemas agrícolas imperantes en el partido de Tandil, con alto grado de insumos y uso de transgénicos que permiten altos rendimientos. Se concluye que la agricultura ecológica, conforma una alternativa a los sistemas convencionales de producción, basadas en sistemas más diversificados y autosuficientes.

Palabras clave: Agriculturización. Agroecología. Indicadores de Sustentabilidad. Sustentabilidad Ambiental.

ABSTRACT: In Argentina, in recent decades, a process known as "agriculturization" has been generated with various environmental impacts. This has led to introducing more sustainable farming models. This paper aims to analyze the sustainability of a rural facility dedicated to organic farming in comparison with conventional agricultural systems located in Tandil, Buenos Aires Province.

The applied methodology is based on the use of sustainability indicators, background papers, interviews, a weaknesses-threats- strengths-opportunities matrix, and suggested corrections of critical points. It is noted that the facility with organic practices, unlike those using conventional practices, has many benefits 
such as high biodiversity, diversification of activities, organic farming, and self-sufficiency, among others. The critical points are reflected in the threats and weaknesses of the system, such as soil usage for sowing and average productivity in crops, being lower than the systems analyzed at the regional level, with high inputs and GMOs use which allow high yields. It is concluded that organic farming is an alternative to conventional production systems based on more diversified and self-sufficient systems.

Keywords: Agriculturization. Agroecology. Sustainability Indicators. Environmental Sustainability.

\section{INTRODUCCIÓN}

El desarrollo económico y social de una región y del país está directamente relacionado con la disponibilidad de sus recursos naturales. Además, toda visión de desarrollo integrado y sostenible a través del tiempo requiere que esos recursos naturales, y especialmente las tierras, mantengan su integridad física y capacidad productiva, en un marco de estabilidad ambiental y social (CISNEROS et al., 2008).

En Argentina, desde fines del siglo XIX, el desarrollo económico estuvo fuertemente vinculado con el sector agropecuario (Chiriboga y Plaza, 1993). LAPITZ et al., (2004) señalan que los países del Cono Sur avanzan desde 1990 hacia la especialización en la producción de oleaginosas, esta tendencia es dramática en Argentina, donde el área dedicada a oleaginosas aumenta desde la década de 1970 en un $382 \%$. Dicho cambio se caracteriza por un paulatino aumento del doble cultivo cereal-soja en detrimento de la disminución de la actividad ganadera. E1 crecimiento de la soja, tanto en superficie sembrada como en rendimiento, ha sido permanente desde su introducción en la región pampeana, el cultivo de esta oleaginosa cobró un nuevo salto en Argentina a partir de 1996, con el lanzamiento al mercado de variedades de soja transgénica (en particular de la soja RR resistente al herbicida glifosato) y su asociación con la siembra directa, la cual intensifica la productividad mediante procesos complejos, estas innovaciones, conocidas como "revolución verde" que se basaron en la utilización de insumos externos de agroquímicos (fertilizantes y pesticidas), en la mecanización de las actividades y en el mejoramiento genético de los cultivos, y se lograron mayores índices de producción agrícola en los agroecosistemas (ALTIERI, 2002). No obstante, esta tecnología generó nuevos problemas (SOLBRIG, 1999), transformando el sistema de producción tradicional a un fenómeno denominado de "agriculturización permanente", con un espectacular avance del cultivo de soja sobre los demás productos agrícolas y ganaderos (PARUELO et al., 2005).

Así, los agroecosistemas pampeanos han sido difusa e intensamente transformados como consecuencia de los procesos agropecuarios. Sus ecosistemas nativos manifiestan un nivel de degradación alto. Por ello, el bioma del pastizal pampeano, y la región ecológica que conforma, acusan la mayor degradación del país (GHERSA y MARTINEZ-GHERSA, 1991).

Además, los agroecosistemas se ven afectados por el uso de agroquímicos y la siembra directa, que han sido parte de los modelos productivos aplicados en la región y, por lo tanto es esperable que hayan impactado sobre funciones de los sistemas agrícolas actuales. Durante los últimos 50 años, los pesticidas se han convertido en uno de los componentes principales de la agricultura (MATSON et al., 1997; HOOPER et al. 2005) y, aunque su uso es considerado económicamente rentable para la mayoría de los sistemas, los efectos secundarios que tienen sobre el ambiente son frecuentemente negativos. Por lo tanto, generan costos que raramente son considerados en los balances para el cálculo de la rentabilidad de la empresa agropecuaria (PIMENTEL, 1992; WILSON y TISDELL, 2001).

Lo anterior estimula a que en la esfera sociocultural, se produzca erosión del conocimiento agrícola ancestral, porque se ignora aquella coevolución que seha producido entre la agricultura local y sus poblaciones originarias. Es de gran importancia el mantenimiento de la diversidad cultural para el sostenimiento de la diversidad biológica, asegura Sarandón et al. (2006), ya que no puede cultivarse lo que no se conoce. En lo económico, la racionalidad se manifiesta en la maximización de la ganancia en 
el corto plazo, con explotación de la naturaleza y del trabajador, y concentración del poder económico (LEFF, 2004). En lo ecológico y social, Altieri (1999) manifiesta que la agricultura a partir de la revolución verde trajo aparejado problemas ambientales tales como erosión del suelo, contaminación por plaguicidas, salinización, problemas sociales (eliminación del predio familiar; concentración de la tierra, los recursos y la producción; crecimiento de la agroindustria y su dominio sobre la producción agrícola; cambio en los patrones de migración rural/ urbana) y uso excesivo de los recursos naturales.

Por lo anterior, es que surge el interés del análisis de la sustentabilidad; de los agroecosistemas, lo cual preocupa a diversos autores (SARANDÓN et al., 2002; VIGLIZZO et al., 2006; VAZQUEZ et al., 2012, VAZQUEZ y ZULAICA, 2013, 2014; entre otros).

En principio un aumento de la sustentabilidad de un proceso productivo implicaría una disminución en los impactos que ésta genera al ambiente. Este análisis implica, no sólo realizar una enumeración de los impactos, sino evaluar la intensidad, magnitud, reversibilidad de los mismos (VIGLIZZO et al., 2003).

Luego, se observa que mientras que el principal objetivo de la agricultura convencional es la obtención de una gran producción en forma de monocultivo y con una ganancia económica significativa, la producción del alimento natural, en armonía con la naturaleza, se basa en la diversificación agroproductiva, y su objetivo principal es la subsistencia del grupo familiar con una modesta inversión de tiempo, con un alimento sano y que produzca mínimos impactos en el medio ambiente. Para esto, Fukuoka (1978) propone una agricultura ecológica, siendo esta según él, la que menor labor física requiere y la más efectiva una vez establecido el balance natural y comprensión básica de cómo funciona el nicho local.

Por lo tanto, una herramienta práctica para medir la sustentabilidad en los agroecosistemas son los indicadores, definidos estos como variables seleccionadas y cuantificadas que nos permite ver una tendencia que de otra forma es no es fácilmente detectable (SARANDÓN y FLORES, 2009).
Luego, el objetivo de este trabajo es analizar la sustentabilidad de un establecimiento rural dedicado a la agricultura ecológica con respeto a los sistemas agrícolas convencionales, ubicados en el partido de Tandil, provincia de Buenos Aires.

\section{Área de Estudio}

El establecimiento seleccionado, presenta características únicas de producción en el partido de Tandil, tal como las mencionadas prácticas agrícolas ecológicas propuestas por Fukuoka (1978); es por esto que se determina el estudio de dicho agroecosistema, debido a que el mismo, se haya inserto en el marco de un modelo productivista, donde la racionalidad ambiental se basa en el búsqueda del máximo beneficio en el corto plazo, sin contemplar los efectos nocivos en lo social y natural (LEFF, 2004).

El partido de Tandil, se localiza en el sector sudeste de la provincia de Buenos Aires, Argentina, y ocupa una superficie de 493.500ha. Limita con los partidos de Rauch, Ayacucho, Balcarce, Lobería, Necochea y Benito Juárez. Según el último censo de población realizado en 2010, el Partido posee una población de 123.343 habitantes, lo cual indica un crecimiento relativo del $14,1 \%$ respecto de 2001 . La mayor parte de la población, habita en la ciudad de Tandil, cabecera del Partido, la cual dista algo más de $300 \mathrm{~km}$ de la ciudad de Buenos Aires y se vincula con el resto de la provincia a través de la Ruta Nacional 226 y las provinciales 30 y 74 (Figura 1 ).

Desde el punto de vista fitogeográfico el área de estudio forma parte de la Provincia Pampeana descripta por Cabrera y Willink (1973). Los autores citados definen como tipo de vegetación dominante la estepa o pseudoestepa de gramíneas, que forman matas de $60 \mathrm{~cm}$ a $1 \mathrm{~m}$ de altura. Siendo el Orden predominante de suelos del partido de Tandil es el Molisol (según la clasificación del Atlas de Suelos de la República Argentina) dado que ocupa el 99\% de la superficie total, que es de $4.935 \mathrm{~km}^{2}$ (SAGYPINTA, 1989). 
Figura 1- Localización del establecimiento rural dedicado a agricultura ecológica. Partido de Tandil. Provincia de Buenos Aires. Argentina.

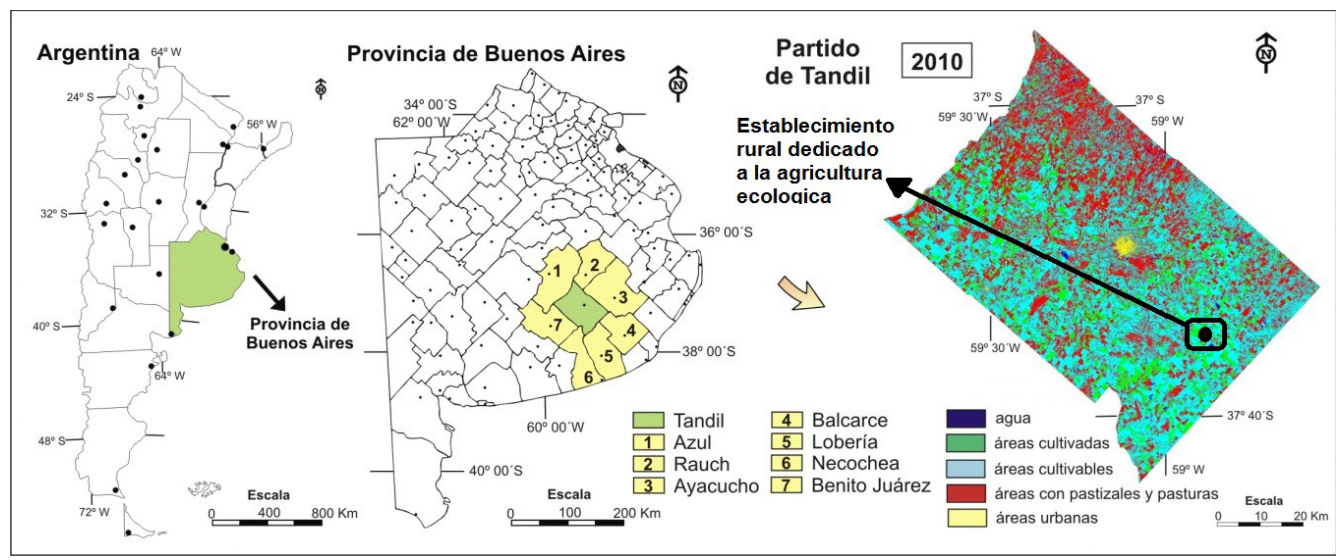

Fuente: Elaboración propia a partir de trabajos antecedentes sobre imágenes

clasificadas Landsat 5 (Vazquez y Zulaica, 2011; 2012).

La circulación regional de la atmósfera es un importante factor del clima en la provincia de Buenos Aires. Su territorio comprende el espacio más frecuente transitado por las masas de aire que se desplazan en dirección NE - SW, dirección predominante tanto en el semestre cálido como en el frío del año Luego, el clima regional es de tipo templado húmedo con influencia oceánica, inviernos suaves y veranos cortos y frescos (BURGOS, 1968). El régimen de precipitaciones presenta un promedio anual de $900 \mathrm{~mm}$ con una distribución normal y una mayor concentración en el período primaveroestival (Centro Regional de Agrometeorología, FAAUNICEN). Se hace necesario tener en cuenta en este punto que la expansión agrícola actual aumenta los riesgos económicos por su elevada dependencia de las condiciones meteorológicas (VAZQUEZ, 2013).

Los ríos del sur de la provincia de Buenos Aires, forman pequeñas redes hidrográficas regidas por las alturas de Tandilia y Ventania, que originan una serie de cuencas que en algunas oportunidades se vinculan con el océano Atlántico, y en otras se pierde por infiltración sin llegar a él, formando lagunas (GABELLONE et al., 2003).

Sánchez et al. (1999) definen al partido en tres compartimentos ecológicos (Serranías, Llanura periserrana y Llanura distal). El compartimento de las Serranías asocia diferentes elementos del sistema orográfico de las Sierras Septentrionales de la provincia de Buenos Aires (Tandilia). Al paisaje serrano le suceden las Llanuras periserranas donde se integran lomadas relativamente bajas y sectores inferiores de faldeos, que poseen buena aptitud para el desarrollo agrícola. El compartimento de la Planicie distal o deprimida sucede altimétricamente a la Llanura periserrana. Está conformado por paisajes de relieve plano, a veces muy achatados y otras veces ligera y uniformemente inclinados.

Según trabajos antecedentes de Vazquez y Zulaica $(2012$; 2011) este partido ha sufrido una transformación respecto al proceso de agriculturización, en el período 1988-2010, donde se observa un incremento de la agricultura del 39,4\%, mientras que las áreas destinadas al pastoreo se redujeron en un $22,8 \%$ (Figura 1). Para los estudios antecedentes fueron realizadas 30 entrevistas de campo donde se obtuvieron datos acerca de los cultivos y prácticas agrícolas realizadas, además de datos obtenidos por informantes calificados, INTA, CREA, Cooperativa Agrícola, entre otros, y se pudo observar que los establecimientos mostraban cambios principalmente a partir del 2002 (VAZQUEZ, 2004) donde la tendencia consiste en la homogeneización de los paisajes agrícolas, transformándose de un proceso mixto agrícola-ganadero, a solamente 
agricultura, principalmente con la introducción de paquetes tecnológicos (soja-glifosato y siembra directa); y con cambios en lo referido a la mano de obra, la cual fue reemplazada por menor cantidad de empleados y con mayor capacitación.

Sin embargo, contrariamente a lo que estaría sucediendo a nivel regional, el establecimiento que se propone analizar en este estudio de caso, muestra tendencias completamente diferenciadas, debido a que en mismo se realiza no solo actividad agrícola (trigo, maíz, cebada, sorgo), sino también actividad ganadera sobre pasturas artificiales y naturales (vacas, cerdos y ovejas), y se diversifica aún más el sistema, adicionando huerta, frutales, procesamiento de trigo en harina, entre otras actividades.

\section{METODOLOGÍA}

La metodología aplicada se basa en el uso de indicadores de sustentabilidad, planteados por Sarandón et al. (2006) y Sarandón y Flores (2009), y aplicados sobre un establecimiento rural dedicado a la agricultura ecológica, propuesta por Fukuoka (1978), este establecimiento se presenta hasta la actualidad como único caso con respecto a las prácticas agrícolas naturales, en detrimento de las convencionales, en el partido de Tandil.

Los indicadores en un principio fueron estandarizados $\mathrm{y}$ ponderados, $\mathrm{y}$ se dividen en: indicadores socio-culturales (ISC), económicos (IK) y ecológicos (IE).

Luego, para evaluar la esfera sociocultural se utilizaron los indicadores: satisfacción de las necesidades básicas (A), donde un sistema sustentable es aquél en el cual los agricultores tienen aseguradas sus necesidades básicas. Comprende vivienda, educación, salud y servicios. Integra los subindicadores: vivienda (A1), acceso a la educación (A2), acceso a salud y cobertura sanitaria (A3) y servicios (A4).

Con respecto a la aceptabilidad del sistema de producción (B) parte de que la satisfacción del productor está directamente relacionada con el grado de aceptación del sistema productivo.

Por otro lado, la integración social (C), se evalúa con respecto a la relación con otros miembros de la comunidad.

Por último, conocimiento y conciencia ecológica (D), se basa en que el conocimiento y la conciencia ecológica son fundamentales para tomar decisiones adecuadas respecto a la conservación de los recursos. Para la ponderación, se consideraron de mayor peso los indicadores de satisfacción de necesidades básicas y el grado de aceptabilidad del sistema productivo, luego el valor del IK, que evaluó la satisfacción de este objetivo, se calculó como la suma algebraica de sus componentes multiplicados por su peso o ponderación, de la siguiente manera:

$\underline{2[(\mathrm{~A} 1+2 \mathrm{~A} 2+2 \mathrm{~A} 3+2 \mathrm{~A} 4) / 7)+2 \mathrm{~B}+\mathrm{C}+\mathrm{D}]}$

6

El indicador IK se calcula a partir de la autosuficiencia alimentaria (A) el cual está compuesto por dos indicadores: diversificación de la producción (A1), donde se tiene como base que un sistema es sustentable si la producción alimentaria es diversificada y alcanza para satisfacer el nivel nutricional de la familia, y superficie de producción de autoconsumo (A2), donde un sistema es sustentable si la superficie destinada a la producción de alimentos para el consumo es adecuada con relación a los integrantes del grupo familiar. El indicador B, ingreso neto mensual por grupo, tiene en cuenta que el sistema es sustentable si puede satisfacer las necesidades económicas del grupo familiar. El indicador $\mathrm{C}$, riesgo económico, se basa en que un sistema será sustentable si minimiza el riesgo económico, asegurando la estabilidad en la producción para las futuras generaciones. Se consideraron tres aspectos: diversificación para la venta $(\mathrm{C} 1)$; número de vías de comercialización (C2) donde se parte de que la diversificación comercial disminuye el riesgo económico; y por último, dependencia de insumos externos (C3), donde un sistema con una alta dependencia de insumos es insustentable en el tiempo.

Sarandón et al. (2006) consideraron que el indicador más importante, por las características del grupo productivo, es la autosuficiencia alimentaria, por lo que, en la ponderación, se le otorgó el doble de peso que al resto. El valor del IK, se calculó de la 
siguiente manera:

$$
\frac{2((\mathrm{~A} 1+\mathrm{A} 2) / 2)+\mathrm{B}+(\mathrm{C} 1+\mathrm{C} 2+2 \mathrm{C} 3) / 4}{4}
$$

Por último, el indicador IE, sirve para evaluar la dimensión ecológica. Se utilizó para ello, el indicador A, conservación de la vida de suelo, que se basa en que un sistema es sustentable si las prácticas mantienen o mejoran la vida en el suelo. Este agrupa 3 subindicadores: manejo de la cobertura vegetal (A1); rotaciones de cultivos (A2) y diversificación de cultivos (A3). Además, el indicador $\mathrm{B}$, riesgo de erosión, toma como supuesto que un sistema es sustentable si logra minimizar o evitar la pérdida de suelo debido a la erosión (en este caso, hídrica). Se compone de 3 subindicadores: pendiente predominante (B1); cobertura vegetal (B2), la cual le provee al suelo una protección contra los agentes climáticos y al riesgo de erosión; orientación de los surcos (B3). También se utilizó el manejo de la biodiversidad (C); aquí la biodiversidad es importante para la regulación del sistema ya que, entre otras funciones, proporciona hábitat y nichos ecológicos para los enemigos naturales. Sus dos componentes: biodiversidad temporal $(\mathrm{C} 1)$, donde las rotaciones de cultivos en los predios, aumentan la diversidad en el tiempo; y biodiversidad espacial (C2), es la diversidad de cultivos en el espacio.

El IE, se calculó con la siguiente formula, otorgándoseles el mismo peso a los 3 indicadores:

$$
\frac{(\mathrm{A} 1+\mathrm{A} 2+\mathrm{A} 3) / 3+(2 \mathrm{~B} 1+\mathrm{B} 2+2 \mathrm{~B} 3) / 5+(\mathrm{C} 1+\mathrm{C} 2) / 2}{3}
$$

Los indicadores fueron estandarizados $\mathrm{y}$ ponderados en intervalos desde 0: no sustentable a 4: muy sustentable, siendo los intervalos considerados para este trabajo los siguientes: muy alto: $4-3,5$ puntos; alto: 3,4 - 2,5 puntos; medio: 2,4 - 1,5 puntos; bajo: 1,4 - 0 puntos.

Luego, se obtuvieron para este trabajo preciso, datos a partir de observación directa y entrevistas semiestructuradas (MUNARRIZ,1992), a 10 productores agropecuarios, que cuentan con establecimientos rurales que poseen ciertas características físicas ambientales similares al establecimiento donde se aplican las técnicas de Fukuoka (1978) (ejemplo: aproximadamente tamaño de 100ha, pendientes equivalentes, es decir campos ubicados en área de llanura periserrana, y campos que son manejados por el productor, no arrendados, entre otras). En estas entrevistas, sobre todo se indago en el tipo de manejo agrícola y ganadero, uso de agroquímicos, pendientes, tipo de suelos, tipo de maquinarias, diversidad de actividades rurales, ingresos anuales, personal, autosuficiencia alimentara, necesidades básicas satisfechas, conciencia ecología, integración, biodiversidad.

Además de realizar una entrevista en profundidad y también a partir de observación directa al "establecimiento rural dedicado a la agricultura ecológica" (FUKUOKA, 1978), las entrevistas en todos los casos realizadas a informantes calificados fueron grabadas y volcadas a planillas.

Por último, se elabora una matriz DAFO con el propósito de obtener un diagnóstico de la situación del territorio, de sus aspectos favorables y desfavorables, de origen interno o externo. Las debilidades son aquellos aspectos negativos internos, mientras que las amenazas son externas. Las fortalezas y oportunidades son los aspectos positivos, pero las primeras son de carácter interno y las segundas externas (CAMARERO, 2006). Ambas metodologías combinadas contribuirán a una comprensión más integral de la situación ambiental del establecimiento destinado a agricultura ecológica.

Con todo lo anterior, y a partir de trabajos antecedentes (VAZQUEZ y ZULAICA, 2012, 2011), además de entrevistas a informantes calificados, y datos obtenidos a partir de observación directa, se analizan los valores de los indicadores, y sus posibles puntos críticos a contemplar, proponiendo a futuro recomendaciones, correcciones y/o monitoreos. Tal como expresan Sarandón y Flores (2009) donde manifiestan que este es el objetivo de la metodología propuesta, luego, el análisis de los indicadores permitirá obtener los puntos donde se presenta mayor insustentabilidad en el caso de estudio. Esto se aprecia como la diferencia entre el valor ideal y el valor real obtenido. 


\section{RESULTADOS}

Primeramente y como fue mencionado en párrafos precedentes, se realizó el análisis de los indicadores de sustentabilidad propuestos para este trabajo, y luego se efectuó una matriz DAFO, la cual conllevara a obtener los puntos críticos, basados en las amenazas (factores internos) y debilidades (factores externos) que presente la matriz respecto del establecimiento con prácticas ecológicas, y las posibles modificaciones o propuestas tendientes a una mayor sustentabilidad del sistema.

Indicadores de sustentabilidad aplicados al establecimiento rural ecológico y establecimientos con prácticas convencionales

Los resultados de los indicadores de sustentabilidad socio-culturales (ISC), como los económicos (IK) y los ecológicos (IE), muestran que la actividad agroecológica en el caso del estudio seleccionado, basado en la técnica de FUKUOKA (1978), presentan valores de muy alta y alta sustentabilidad. Mientras que los productores entrevistados en estudios antecedentes de los cuales se observa la media para los mismos, se muestran valores de baja a media la sustentabilidad (Figura 2).

Figura 2 - Indicadores de sustentabilidad para el establecimiento ecológico respecto de los establecimientos a nivel regional. Partido de Tandil.

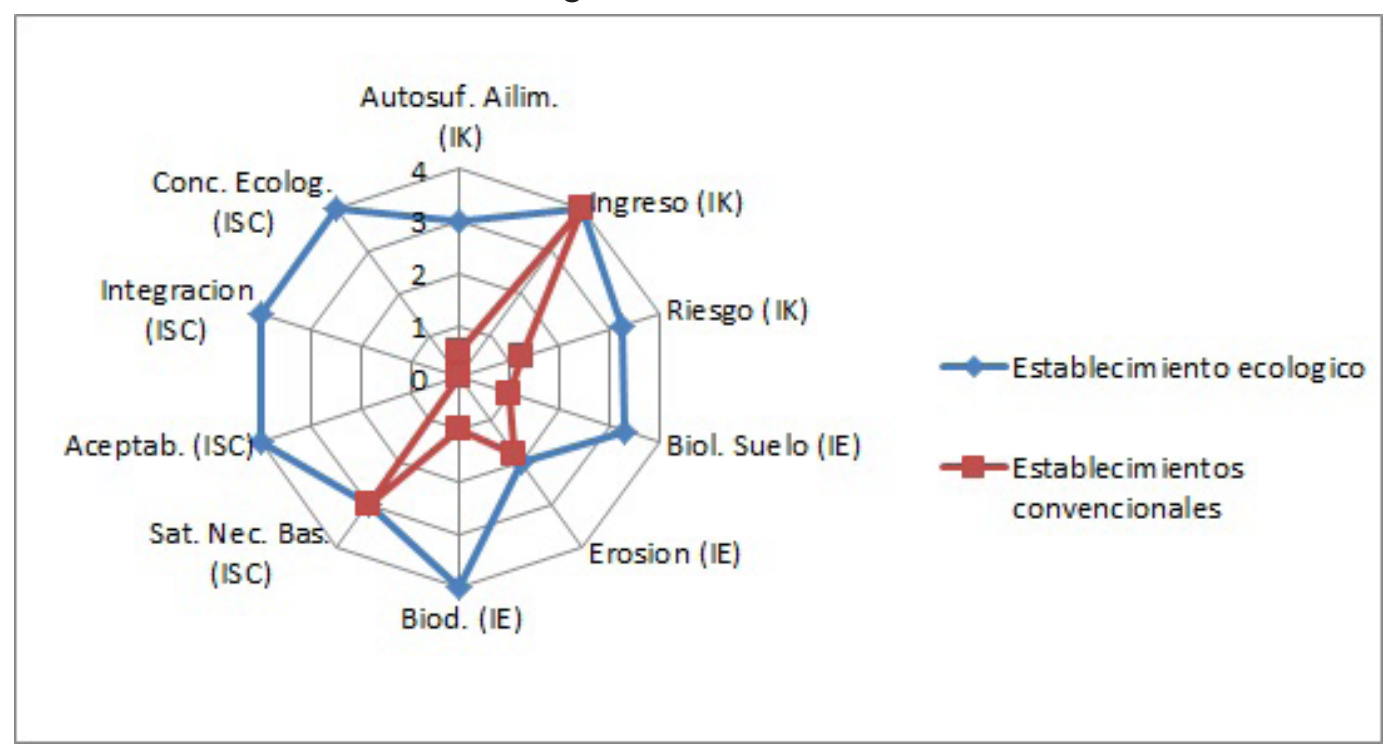

Fuente: Elaboración propia a partir de datos obtenidos en entrevistas y estudios antecedentes (VAZQUEZ y ZULAICA, 2011; 2012).

A continuación se describe cada indicador, con sus respectivo subindicadores en el caso de que así sea:

\section{Indicador de Socio-Cultural (ISC)}

Con respecto al indicador SC se observó que el punto A) referido a la satisfacción de las necesidades básicas, mostró que la vivienda era de 
material terminada, la educación con acceso a escuela secundaria, en el caso de la salud presentó centro sanitario con médicos permanentes e infraestructura adecuada, y con respecto a los servicios cuenta con instalación de luz y agua de pozo. Mientras que el punto B) referido a la aceptabilidad del sistema de producción demostró que el productor está muy conforme con lo que hace, y que no haría otra actividad aunque ésta le reporte más ingresos. El punto C) presentó una integración social muy alta. $\mathrm{Y}$ por último el punto $\mathrm{D}$ ) referido al conocimiento y conciencia ecológica mostró que el productor concibe la ecología desde una visión amplia, más allá de su finca y conoce sus fundamentos.

Ahora bien, si se analiza a nivel de casos de estudios de trabajos antecedentes se observa que: el punto A) referido a la satisfacción de las necesidades básicas expuso las mismas características que en el caso anterior. Mientras que el punto B) referido a la aceptabilidad del sistema de producción demostró que los productores están desilusionados con la vida que llevan, que no realizarían más esta actividad. Los mismos, están esperando que se le presente una oportunidad para dejar la producción. El punto C) presentó una integración social media. Por último, el punto D) referido al conocimiento y conciencia ecológica mostró que el productor no presenta ningún tipo de conciencia ecológica, y realiza una práctica agresiva al medio por causa de este desconocimiento.

Luego, se puede observar que ISC para el área de estudio tiene un valor muy alto $(3,66)$, correspondiente al intervalo entre 4 y 3,5; el cual indica una muy alta sustentabilidad. Mientras que a nivel regional el ISC presento un valor de 1, mostrando baja sustentabilidad, encontrándose en el intervalo de 0 a 1,4 .

\section{Indicador de Económico (IK)}

Con respecto al indicador $\mathrm{K}$ los resultados fueron, con respecto al punto A) referido a la autosuficiencia alimentaria, y conformado por la diversificación de la producción, se observó que en el establecimiento con prácticas ecológicas, alcanzó valores mayores a 9, y que la superficie de producción de autoconsumo que varía entre 10 y 25ha. Con respecto al punto B), referido al ingreso neto mensual es mayor a 25000 pesos mensuales. Y por último, haciendo referencia al punto $\mathrm{C}$ ), de riesgo económico, se divide en tres puntuaciones, entre ellos diversificación de la venta que llega a más de 6 productos (trigo, harina, vacas, cerdos, ovejas, verduras, entre otros), el número de vías de comercialización que llega a más de 5 canales (dietéticas, particulares, verdulerías, carnicerías, mercados, entre otros) y la dependencia de insumos externos que es la baja de 0 a $20 \%$.

Ahora bien, si analizamos a nivel regional, se obtiene que el punto A) referido a la autosuficiencia alimentaria, y conformado por la diversificación de la producción presentó valores entre 3 y 4 en la diversificación de producción, y la superficie de producción de autoconsumo es menor o igual a 1 ha. Con respecto al punto $\mathrm{B}$ ), referido al ingreso neto mensual es también mayor a 25000 pesos mensuales. $Y$ por último, haciendo referencia al punto $\mathrm{C}$ ), de riesgo económico, el cual se divide en tres puntuaciones, entre ellos diversificación de la venta que llega tan solo a 3 o 4 productos (cereales/oleaginosas), el número de vías de comercialización que llega a 3 canales (cooperativas agrícolas; molinos; aceiteras) y en este caso la dependencia de insumos externos es la más alta, del 80 a $100 \%$.

Luego, se puede observar que el indicador IK para el área de estudio seleccionada, correspondiente a la práctica de Fukuoka (1978) tiene un valor alto de sustentabilidad $(3,31)$, correspondiente al intervalo entre 2,5 y 3,4 . Mientras que a nivel regional el IK se caracterizó por valores de sustentabilidad medio 1,62 , en el intervalo de 1,5 a 2,4 .

\section{Indicador de Ecológico (IE)}

Con respecto al indicador ecológico (IE), a nivel del establecimiento rural seleccionado, en el punto A) referido a conservación de la vida de suelo, el cual se encuentra conformado por tres subindicadores, mostró que el manejo de la cobertura vegetal se corresponde a valores del 50 a $75 \%$, además se rotan los cultivos todos los años, y se deja 
descansar un año el lote e incorpora leguminosas o abonos verdes, conjuntamente el establecimiento es totalmente diversificado, con asociaciones de cultivos y con vegetación natural.

El punto B) referido a riesgo de erosión que se divide en los subindicadores: pendiente predominante, donde se observó que la misma es de 3 y $5 \%$ (SANCHEZ y ZULAICA, 2002), cobertura vegetal en la cual se destacaron valores del 50 al 74\% y orientación de los surcos, siendo para este caso de estudio, perpendiculares a la pendiente.

Con respecto al manejo de la biodiversidad, punto C), se observó que con respecto a biodiversidad temporal se realizan rotaciones todos los años, se deja además descansar un año el potrero o incorpora leguminosas o abonos verdes; y con respecto a la biodiversidad espacial el establecimiento es totalmente diversificado, con asociaciones entre ellos y con vegetación natural.

El indicador ecológico (IE), a nivel de los establecimientos rurales característicos de la región, en el punto A) referido a conservación de la vida de suelo, mostró que el manejo de la cobertura vegetal, alcanza valores del 25 a $50 \%$, realiza rotaciones eventualmente, y además posee poca diversificación de cultivos, sin asociaciones.

El punto B) referido a riesgo de erosión que se divide en los subindicadores: pendiente predominante, donde se observó que la misma es de 3 y $5 \%$, cobertura vegetal en la cual se destacaron valores del 25 al $49 \%$ y orientación de los surcos, siendo la media para los demás establecimientos a comparar con el caso de estudio, surcos orientados con $60^{\circ}$ respecto a la pendiente.

Con respecto al manejo de la biodiversidad, punto C), se observó que en cuanto a la biodiversidad temporal se realizan rotaciones eventualmente; y desde la biodiversidad espacial, presentó poca diversificación de cultivos, sin asociaciones.

Luego, se puede observar que el indicador IE para el área de estudio seleccionada, correspondiente a la práctica de Fukuoka (1978) tiene un valor alto de sustentabilidad $(3,11)$, correspondiente al intervalo entre 3,4 y 2,5. Mientras que a nivel regional el $\mathrm{K}$ se caracterizó por valores de sustentabilidad medios 1,26 , en el intervalo de 1,5 a 2,4.

\section{Matriz DAFO}

A continuación en la Tabla 1 se observan las Debilidades, Amenazas, Fortalezas y Oportunidades del establecimiento ecológico propuesto por Fukuoka (1978) con respeto a los sistemas regionales del partido de Tandil. 
Establecimiento agroproductivo ecologico vs. agricultura convencional: Partido de Tandil, Provincia de Buenos Aires Patricia Vasquez, Milagros Vignolles

Tabla 1 - Análisis de la Matriz DAFO del establecimiento ecológico respecto de los establecimientos convencionales a nivel regional.

\begin{tabular}{|c|c|}
\hline Debilidades & Amenazas \\
\hline \multirow[t]{4}{*}{$\begin{array}{l}\text { La productividad de los cultivos del establecimiento ecológico, es menor } \\
\text { que la media de los sistemas agrícolas imperantes en el partido de Tandil, } \\
\text { los cuales se ven afectados por el uso de agroquímicos, transgénicos y la } \\
\text { siembra directa, con altos rendimientos. Tal como expresan MATSON et } \\
\text { al., (1997) y HOOPER et al. (2005) que durante los últimos } 50 \text { años, los } \\
\text { pesticidas se han convertido en uno de los componentes principales de } \\
\text { la agricultura; estos generan costos que raramente son considerados en } \\
\text { los balances para el cálculo de la rentabilidad de la empresa agropecuaria } \\
\text { (Pimentel, 1992; Wilson y Tisdell, 2001). }\end{array}$} & $\begin{array}{l}\text { La conservación de suelos en el establecimiento ecológico, mostró } \\
\text { que si bien el manejo es adecuado (con descanso de suelos, siembra } \\
\text { perpendicular a las pendientes e incorporación de abonos verdes, que } \\
\text { aumentan la fertilidad y retención de la capa superior del suelo), se hace } \\
\text { necesario fortalecer este punto, debido a las pendientes y consecuente } \\
\text { erosión hídrica en el área, ya que la técnica consiste en arar la tierra y } \\
\text { sembrarla. Mientras que en los sistemas agrícolas convencionales se } \\
\text { propone la siembra directa que mejora la retención de la capa superior } \\
\text { del suelo, la cual queda protegida por los rastrojos, aunque esta técnica } \\
\text { implica altas dosis de herbicidas (glifosato), sumado al doble cultivo } \\
\text { anual (Vazquez, 2004). }\end{array}$ \\
\hline & $\begin{array}{l}\text { Posibilidad de perdurabilidad del sistema agrícola ecológico dentro del } \\
\text { modelo de producción intensivo a nivel regional. }\end{array}$ \\
\hline & $\begin{array}{l}\text { Falta de incentivos y asistencia técnica, por parte de las autoridades y } \\
\text { organizaciones. }\end{array}$ \\
\hline & $\begin{array}{l}\text { Las investigaciones financiadas por el Estado al igual que las medidas } \\
\text { económicas y políticas impulsadas por él, contribuyen a los sistemas } \\
\text { convencionales en desmedro de este tipo de emprendimientos. }\end{array}$ \\
\hline Fortalezas & Oportunidades \\
\hline $\begin{array}{l}\text { Alta biodiversidad del establecimiento ecológico, se observan diversos } \\
\text { cultivos, además de vegetación natural y pasturas artificiales destinados a } \\
\text { pastoreo de animales. Permitiendo además el autoabastecimiento. Mientras } \\
\text { que respecto de los sistemas productivos del Partido en general, casi todo } \\
\text { lo producido (en un } 80 \% \text { ) se exporta a otros países, el resto es derivado a } \\
\text { molinos y aceiteras nacionales. TEUBAL (2006) expresa que existe una } \\
\text { creciente pérdida de la soberanía alimentaria; gran vulnerabilidad del } \\
\text { agro a causa de la especialización de cultivos exclusivamente destinados } \\
\text { a exportación. }\end{array}$ & $\begin{array}{l}\text { Posibilidad de aplicar certificación de productos, tal como lo propone } \\
\text { el listado de las GLOBAL GAP (2013), lo cual permite el logro del } \\
\text { desarrollo de buenas prácticas agrícolas y un valor agregado a productos } \\
\text { sanos (Vazquez y Zulaica, 2014). }\end{array}$ \\
\hline $\begin{array}{l}\text { La diversificación en la producción permite disminuir el riesgo económico. } \\
\text { No siendo el caso de los sistemas agrícolas imperantes en el partido de } \\
\text { Tandil, ya que la mayoría dependen principalmente del monocultivo } \\
\text { (Glycine max). Además la baja dependencia de insumos externos, } \\
\text { minimiza los costos y reduce el riesgo económico y ambiental, ya que } \\
\text { queda libre de contaminación por agro-tóxicos (alta conciencia ecológica). } \\
\text { Lo que se contrapone a las prácticas regionales (desconocimiento } \\
\text { ambiental). }\end{array}$ & $\begin{array}{l}\text { La experiencia en este predio podría ser compartida hacia el resto } \\
\text { de la productores agropecuarios, propulsando así prácticas de mayor } \\
\text { sustentabilidad, un mayor respeto por el medio ambiente y una } \\
\text { alimentación más sana. }\end{array}$ \\
\hline $\begin{array}{l}\text { Alta aceptabilidad del sistema, además de alta integración social, en } \\
\text { el establecimiento ecológico, lo que difiere de los establecimientos a } \\
\text { nivel regional, ALTIERI (1999) manifiesta que la agricultura a partir } \\
\text { de la revolución verde trajo aparejado problemas sociales tales como } \\
\text { eliminación del predio familiar; concentración de la tierra, cambio en } \\
\text { los patrones de migración rural/urbana y uso excesivo de los recursos } \\
\text { naturales. }\end{array}$ & \\
\hline
\end{tabular}

Fuente: Elaboración propia. 
La matriz DAFO (Tabla 1), permite obtener un diagnóstico preliminar del establecimiento rural con practicas ecológicas, a partir de observar sus fundamentales amenazas y debilidades, como así también las oportunidades y fortalezas que brinda el agroecosistema, esto contribuye a comprender la situación actual del establecimiento rural ecológico, y permite posteriormente presentar medidas correctivas de los puntos críticos, tal como lo proponen Sarandón y Flores (2009); en este caso definidos dichos puntos, como aquellas amenazas y debilidades que se presentan en la matriz DAFO, con la expectativa de finalmente lograr efectuar un monitoreo y/o propuestas para mitigar los efectos negativos de estos puntos críticos en el tiempo, pudiendo así disminuir los riesgos que presentan en el establecimiento, para asegurar la perdurabilidad en el tiempo del mismo.

Luego, y ante lo expresado en la DAFO, se observa que en cuanto a las debilidades internas del sistema, respectodela producciónmedia delos cultivos en función de los demás establecimientos con altos insumos, y por ende con altos rendimientos, se podría analizar la incorporación de mayores abonos verdes con la finalidad de obtener mejoras en el fertilidad del suelo, además de la consecuente retención de la capa superior del suelo, esto debido a que las pendientes donde se encuentran los establecimientos rurales ( 3 y 5\%), correspondientes al área periserrana (muy ondulada), es donde dominan las geoformas modeladas por la acción hídrica (SANCHEZ et al., 1999), esto provoca perdida de materia orgánica, conjuntamente con nutrientes (nitrógeno, fósforo, potasio, entre otros), hacia zonas más bajas. Por lo tanto, sería fundamental realizar monitoreos anuales a partir de estudios de suelos para analizar el estado del mismo, además de sembrar en base a curvas de nivel, e implementar barreras forestales que corten las pendientes. Los árboles y/o arbustos cumplen con muchos propósitos como producción (madera, leña, forraje, frutas, medicinas) además de servicios (sombra para cultivos y/o animales, protección como en el caso de cortinas rompevientos), conjuntamente, los árboles aumentan la diversidad biológica del agroecosistema creando en sus ramas, en sus raíces y en la hojarasca, hogares para otros organismos
(BEER et al., 2004). Fukuoka (1978) propone en estos casos a las leguminosas, tal como las acacias, porque su rápido crecimiento y fijación de nitrógeno en el suelo. También sería de gran importancia conservar la vegetación natural del área, que permite tener una alta biodiverdidad, con una consecuente importante riqueza del agroecosistema.

Mientras que en cuanto a las amenazas externas expuestas en la DAFO (Tabla 1), si bien los sistemas regionales no se condicen con las practicas agropecuarias convencionales, eso hace pensar en que la posibilidad de perdurabilidad del sistema agrícola ecológico dentro del modelo de producción intensivo tendería a ser débil respecto del resto, sin embargo, se observa que la diversificación de actividades hace posible el autoabastecimiento, sin especular en grandes ganancias, aunque más de una vez sobrepasando los ingresos anuales de los establecimientos con prácticas convencionales.

Asimismo, conrespecto a la falta de incentivos y asistencia técnica, por parte de las autoridades y organizaciones, el análisis y estudio de este sistema de producción plasmado en la realidad puede generar interés y convocar a científicos, ONG's, entre otras instituciones, con la finalidad de propulsar las medidas económicas y políticas impulsadas a partir de un ordenamiento territorial que permita contribuir a este tipo de emprendimientos con agricultura ecológica en desmedro de los sistemas convencionales, y así lograr una mayor sustentabilidad ambiental a nivel regional.

\section{DISCUSIÓN FINAL Y CONCLUSIONES}

Se observa respecto del objetivo propuesto que en el caso de estudio seleccionado con prácticas agrícolas ecológicas propuestas por Fukuoka (1978), el mismo presenta condiciones de sustentabilidad más favorables respecto a los sistemas agrícolas convencionales que se encuentran en el partido de Tandil, provincia de Buenos Aires, Argentina.

Esto surge de observar los indicadores de sustentabilidad aplicados al caso de estudio con prácticas agrícolas ecológicas, los cuales muestran mejores condiciones de sustentabilidad con respecto a los tres indicadores analizados, 
tanto los socio-culturales, como los económicos y ecológicos, siendo los valores para el primero muy alto y para los últimos dos alto. Mientras que en lo que se refiere a lo regional se observan diferencias respecto a la sustentabilidad de los establecimientos convencionales seleccionados, dado que presentan valores significativamente menores (bajos), en lo referido al punto socio-cultural, y presentando valores medios para los indicadores económicos y ecológicos.

En base al análisis de la situación de los predios y de los resultados de los indicadores de sustentabilidad, se construyó una matriz DAFO la cual permitió determinar aquellas fortalezas, amenazas, oportunidades y debilidades de cada tipo de agroecosistema. Sobresaliendo en esta instancia múltiples fortalezas en el caso del establecimiento con prácticas correspondientes a la agricultura ecológica propuesta por Fukuoka; lo cual se debe a las características particulares que este tipo de agricultura propulsa, donde se realiza una mayor diversificación de cultivos, lo que representa beneficios no solo a nivel ecológico, ya que permite la conservación de la vida del suelo y de la biodiversidad, sino que además disminuye el riesgo económico debido a la diversificación para la venta. Por lo cual, se diferencia de los sistemas agrícolas imperantes en el partido, ya que estos incorporan la realización del doble cultivo anual (cereal/oleaginosa), lo que genera impactos como simplificación del ecosistema, degradación de suelos, pérdida de biodiversidad, y consecuentemente disminución de la riqueza, entre otros.

Con respecto a las recomendaciones con respecto a los puntos críticos que se establecen en este caso de estudio, a partir de la DAFO, se sugiere principalmente por las pendientes y consecuente erosión hídrica en la zona, incorporar siembra teniendo en cuenta las curvas de nivel, y completar el sistema con barreras de arboledas perpendiculares a las pendientes, esto permitirá un mejor manejo del suelo y como consecuencia mayores rendimientos de los cultivos sembrados.

Se concluye que la agricultura ecológica, conforma una alternativa a los sistemas convencionales de producción, basadas en sistemas más diversificados $\mathrm{y}$ autosuficientes, tal como lo propone la agroecología y en la misma línea, la agricultura natural propuesta por Fukuoka, propulsándose el equilibrio natural y autorregulación de los agroecosistemas y la no dependencia de insumos artificiales, con la posibilidad de aplicar la certificación de los productos agrícolas obtenidos.

\section{REFERENCIAS}

ALTIERI, M. Agroecología. Bases cientificas para una agricultura sustentable. Edit. Nordan-Comunidad. Montevideo. 1999. cap. 17. p: 307.

ALTIERI, M.A. 2002. Los impactos ecológicos de los cultivos transgénicos y las razones por la que la biotecnología agrícola es incompatible con una agricultura sostenible. En: SARANDÓN (ED.). Agroecología. El camino hacia una agricultura sustentable. cap. 11: 223-247. E.C.A.

BEER J.; IBRAHIM, M.; SOMARRIBA, E.; BARRANCE, A.; LEAKEY R. Establecimiento y manejo de árboles en sistemas agroforestales. Cap. 6. Árboles de Centroamérica. OFI CATIE. 46 p. 2004.

BURGOS, J.J. El clima de la Provincia de Buenos Aires en relación con la vegetación natural del suelo. En: Cabrera (ed.) Flora de la provincia de Buenos Aires. Tomo IV. Parte I:3-100. INTA. Col. Científica. 1968.

CABRERA, A.; WILLINK, A. Biogeografia de América Latina. Washington: Secretaría General de la Organización de los Estados Americanos, 1973.

CAMARERO, L. (Coordinador). Medio Ambiente y Sociedad: Elementos de Explicación Sociológica. Editorial Thomson. Madrid. 2006. 384p.

CHIRIBOGA, M.; PLAZA, O. Desarrollo rural microrregional y descentralización. San José de Costa Rica: IICA, 226 p. 1993.

CISNEROS, J.; CHOLAKY, C.; CANTERO GUTIERREZ, A.; GONZALES, J.; REYNERO, M., DIEZ, A.; BERGESIO, L. Erosión Hídrica. Principios 
y técnicas de manejos. Universidad Nacional de Rio Cuarto. Facultad de Agronomía y Veterinaria. Servicio de Conservación y Ordenamiento Territorial de Tierras (SECYOT). Editora UNIRIO. Colección Académico Científico. 2012.

FUKUOKA, M. The one-straw revolution, an introduction to natural farming. Rodale press. Editado por Instituto Permacultura Montsant.1978.

GABELLONE, N.A.; SARANDON, R.; CLAP, C. Caracterización y zonificación ecológica de la cuenca del Río Salado. En: Maiola, Gabellone y Hernández (eds.). Inundaciones en la Región Pampeana: 89 -122. Edulp, Editorial de la Universidad de La Plata. Honorable Cámara de Diputados de la Pcia. de Bs. As. 2003.

GHERSA, C.M.; MARTÍNEZ-GHERSA, M.A. Cambios ecológicos en los agroecosistemas de la Pampa Ondulada; Efectos de la introducción de la Soja. Ciencia e Investigación, Facultad de Farmacia y Bioquímica, UNMSM, 5: 182-188. 1991.

HOOPER, D.; CHAPIN, III S.; EWEL, J.; HECTOR, A.; INCHAUSTI, P. Effects of biodiversity on ecosystem functioning: a consensus of current knowledge. Ecological Monographs. v.75, n.1, 2005. DOI: http://dx.doi.org/10.1890/04-0922

LEFF, E. Racionalidad ambiental. La aprobación social de la naturaleza. Siglo XXI. México. Cap. 5. 2004. p:181-231.

MATSON, P.; PARTON, W.; POWER, A. y SWIFT, M. Agricultural Intensification and Ecosystem Properties. Science 277:504-509. 1997. DOI: 10.1126/ science.277.5325.504

MUNARRIZ IRAÑETA, B. Técnicas y métodos en investigación cualitativa. Universidade da Coruña, Servicio de Publicaciones. 1992. p:101-116.

LAPITZ, R.; EVIA, G.; GUDYNAS, E. Soja y carne en el Mercosur; comercio, ambiente y desarrollo agropecuario. Montevideo: Coscoroba, 303 p. 2004.
PARUELO, J.; GUERSCHMAN, J.; VERÓN, S. 2005. Expansión agrícola y cambios en el uso del suelo, Ciencia Hoy, vol. 15, No 87.

PIMENTEL, D. Ecological effects of pesticides of Non-Target species in terrestrial ecosystems. In: TARDIFF, R.G. (org.) Methods to Assess Adverse Effects of Pesticides on Non-target Organisms. New York: John Wiley \& Sons, 1992.

SAGyP-INTA. Mapa de Suelos de la Provincia de Buenos Aires, Buenos Aires: Ediciones Secretaría de Agricultura Ganadería y Pesca - Instituto de Tecnología Agropecuaria. Proyecto PNUD Argentina 85/019. 1989.

SÁNCHEZ, R.; ZULAICA, L. Ordenamiento Morfoedáfico de los Sistemas Ecologicos-Paisajísticos del Partido de Tandil. Contribuciones Científicas. EGEA. 2002.

SÁNCHEZ, R.; MATTUS, G.; ZULAICA, L., Compartimentación ecológica y ambiental del partido de Tandil (Provincia de Buenos Aires). In: Congreso Ambiental '99. Programa de Estudios Ambientales, Universidad Nacional de San Juan, San Juan, 1999.

SOLBRIG, O.; VIGLIZZO, E. Sustainable Farming in the Argentine Pampas: History, Society, Economy and Ecology. DRCLAS Working Paper n. 99/00-1. Cambridge: Universidad de Harvard, 1999. DOI: 10.1.1.202.7923

SARANDÓN, S.J.; ZULUAGA, M.S.; CIEZA, R.; GÓMEZ, C.; JANJETIC, L.; NEGRETE, E. Evaluación de la sustentabilidad de sistemas agrícolas de fincas en Misiones, Argentina, mediante el uso de indicadores. Agroecología 1: 20-28. 2006.

SARANDON, S.J.; FLORES, C.C. Evaluación de la sustentabilidad en agroecosistemas: una propuesta metodológica. Agroecología. No 4. 2009. p.19-28.

SOLBRIG, O. Observaciones sobre la diversidad y desarrollo agrícola. En: Matteucci, Solbrig, Morello y Halffter (eds.) Biodiversidad y uso de la tierra. 
Conceptos y ejemplos Latinoamérica. Cap 3: 41-54, col C.E.A. 24. 1999.

TEUBAL, M. Expansión del modelo sojero en la Argentina. De la producción de alimentos a los commodities. Realidad Económica. N $^{\circ} 220.2006$.

VAZQUEZ, P. 2004. Comparación temporal de la sustentabilidad de dos modalidades de producción agrícolas (Tandil, Argentina). Tesis de Licenciatura en Diagnóstico y Gestión Ambiental, Facultad de Ciencias Humanas, Universidad Nacional del Centro de la Provincia de Buenos Aires, Tandil, Argentina. $185 \mathrm{p}$.

VAZQUEZ, P. Comparación de índices de estrés hídrico, a partir de información captada por el sensor MODIS, en la Región Pampeana Argentina. Revista Cuadernos Geográficos de la Universidad de Granada. Cuadernos Geográficos, v. 52, n. 1. 2013.

VAZQUEZ, P., ZULAICA, L. Agriculturization and environmental impacts in a representative area of the ecoregion of the Pampas, Argentina. Brazilian Geographical Journal: Geosciences and Humanities research medium, Universidade Federal de Uberlândia, v. 5, n. 1, 2014.

VAZQUEZ, P.; ZULAICA, L. Intensificación agrícola y pérdida de servicios ambientales en el partido de Azul (provincia de Buenos Aires, Argentina) entre 20022010. Revista Sociedade \& Natureza, Universidade Federal de Uberlandia, v. 25, n. 3, 2013. DOI: http:// dx.doi.org/10.1590/S1982-45132013000300008

VAZQUEZ, P.; ZULAICA, L. Transformaciones agroproductivas e indicadores de sustentabilidad en el Partido de Tandil (Provincia de Buenos Aires) durante los períodos 1988-2002 y 2002-2010. Revista Campo-Território: Revista de Geografía Agrária. v.7, n.13, 2012.

VAZQUEZ, P.; ZULAICA, L. Cambios en el uso de la tierra del Partido de Tandil y principales impactos ambientales. Revista Párrafos Geográficos. Editada por el IGEOPAT. n. 2. 2011.
VAZQUEZ, P.; SACIDO, M.; ZULAICA, L. Transformaciones agroproductivas e indicadores de sustentabilidad en la Cuenca del río Quequén Grande, Provincia de Buenos Aires, Argentina) durante los períodos 1988-1998 y 1998-2008. Cuadernos Geográficos, № $50,2012$.

VIGLIZZO, E.F., PORDOMINGO, A.J., CASTRO, M.G. y LÉRTORA, F.A. 2003. Environmental assessment of agriculture at a regional scale in the pampas of Argentina. Environmental Monitoring \& Assessment 87: 169-195.

VIGLIZZO, E.F., FRANK, F., BERNARDOS, J., BUSCCHIAZO, D.E. y CABO, S. 2006. A rapid method for assessing the environmental performance of commercial farms in the pampas of Argentina. Environmental Monitoring \& Assessment 117: 109-134.

WILSON, C. y TISDELL, C. 2001. Why farmers continue to use pesticides despite environmental, health and sustainability costs. Ecological Economics 39:449-462. 